\title{
SIMULACIÓN DEL COMPORTAMIENTO TÉRMICO DE UN PROTOTIPO HABITACIONAL
}

VERA, Luis H.; ZINI, Luciano A.; CÁCERES, Manuel; FIRMAN, Andrés; BUSSO, Arturo (*)

\section{RESUMEN:}

Este trabajo tiene como objetivo principal determinar el comportamiento térmico del prototipo habitacional localizado en el Campus del Valle de la UFRGS - Universidade Federal do Rio Grande do Sul en la ciudad de Porto Alegre, a través de una simulación realizada con el programa computacional Energy Plus. Los valores obtenidos a lo largo de un año de simulación, son comparados estadísticamente y presentados gráficamente en una carta psicométrica para determinar las mejores estrategias de confort. Inicialmente se consideró una vivienda cerrada y vacía un año completo. Seguidamente se simuló la vivienda agregando personas y equipamiento eléctrico para estudiar las condiciones de confort (PMV) para una vivienda de 4 personas. Finalmente se determinó el sistema de aire acondicionado más adecuado para esta edificación.

Palabras clave: Carta Bioclimática, Energy Plus, Simulación Energética.

\section{INTRODUCCION.}

Considerando que el sector de la construcción representa hoy en día el 40\% del consumo energético mundial, esto nos lleva a analizar que en poco tiempo los grandes consumidores de energía serán los edificios, comercios, industrias y viviendas.

Si bien estas nuevas infraestructuras son necesarias para el desarrollo de muchos países, la demanda energética asociada con la mejora de la calidad de vida, así como con el crecimiento económico, crean una relación que, evaluando los modelos existentes, no es compatible con el desarrollo sostenible y, por lo tanto, es necesario vincular este crecimiento con nuevos paradigmas de eficiencia energética y utilización de energías renovables.

En la Argentina, las torres comerciales representan el 19\% del total de la electricidad consumida, las residenciales alcanzan el 21\% y los edificios públicos, el 7\%. Los datos no pasan inadvertidos, sobre todo si

(*) GER - Grupo en Energías Renovables - FaCENA - UNNE. Av. Libertad 5470 - 3400, Corrientes, Argentina. Tel./Fax: (0379) 4473931 int. 129, e-mail: 1h_vera@yahoo.com.ar 
consideramos que la matriz energética local está basada principalmente en fuentes de energías no renovables. En este escenario, la eficiencia energética emerge como uno de los valores que orientan y orientarán cada vez más la construcción

De esta manera, para responder de forma sostenible a las demandas energéticas en las construcciones, se deben crear nuevos sistemas que permitan optimizar las demandas asociada a la iluminación y climatización utilizadas manteniendo adecuadas las condiciones de confort.

Para esto, la simulación juega un papel fundamental si se quiere reducir el consumo energético, ya que es posible estudiar el comportamiento térmico y el consumo energético previamente a la construcción del edificio o a su remodelación, y permite analizar e investigar distintas alternativas de diseño y uso del edificio, comparando soluciones y optando por aquellas de menor consumo. La única forma de tener edificios optimizados desde el punto de vista energético es simulándolos, y es mucho más económico y efectivo que experimentar directamente con distintos edificios modelos (cada uno es diferente y tiene un comportamiento térmico distinto).

Por lo expuesto, el presente trabajo tiene como objetivo analizar las condiciones de confort de una vivienda (denominada Prototipo Alvorada) en la que fueron aplicados criterios bioclimáticos y de sostenibilidad, para evaluar la demanda energética necesaria para mantener las condiciones de confort en su interior.

Para alcanzar el objetivo planteado se ha desarrollado el modelo de una vivienda existente dentro del programa computacional Energy Plus, realizando simulaciones en base horaria se ha evaluado su comportamiento higrotérmico anual y se ha determinado la necesidad de energía necesaria.

\section{Confort Térmico}

Se define Confort Térmico como el estado mental que expresa la satisfacción del hombre con el ambiente térmico que lo rodea. La insatisfacción puede ser causada por la sensación de desconfort por el calor o por el frio, cuando el balance térmico no es estable, es decir, cuando hay diferencias entre el calor producido por el cuerpo y el calor transferido por el mismo al ambiente. La norma internacional que rige el confort térmico en ambientes es la ISO 7730 (2006).

El calor producido en el cuerpo es determinado por el nivel de actividad de la persona, siendo también variable con la edad y el género. Este calor es intercambiado con el medio ambiente por conducción, convección, radiación y evaporación. La conducción generalmente no tiene gran relevancia. La convección depende de la temperatura y la velocidad del aire circundante a la superficie. La radiación depende de la temperatura media radiante y la evaporación depende de la humedad del aire y de su velocidad.

Los parámetros más importantes del confort térmico se subdividen en dos clases:

- Parámetros Individuales: actividad y vestimenta. 
- Parámetros Ambientales: temperatura del aire, humedad del aire, velocidad del aire y temperatura media radiante.

La norma ISO 7730 considera que un espacio presenta condiciones de confort térmico cuando no más del $10 \%$ de sus ocupantes se sientan inconfortables.

La cuantificación del porcentaje de desconfort fue hecha a través de estudios que implicaron 1300 personas. Estos estudios permitieron establecer una relación entre el resultado del balance energético del cuerpo y la tendencia de insatisfacción, llamada como PPD (Predicted Percetage of Dissatisfied) o PMV (voto medio previsto).

La metodología para determinar el PMV de cálculo consiste en los siguientes puntos:

a) Parámetros: se cuantifican los parámetros individuales y ambientales de las personas y del ambiente.

b) Ecuación de confort: se substituyen estos valores en la ecuación de confort térmico para la determinación del término asociado a la acumulación energética en el cuerpo.

c) Voto medio previsto $(P M V)$ : con base en el valor de la acumulación energética en el cuerpo y en el metabolismo se determina el valor del voto medio previsto (Predicted Mean Vote, $P M V$ ) a través de una correlación. El PMV no es más que una escala cuantitativa de la sensación de calor y de frio.

d) Insatisfacción: el porcentaje de personas insatisfechas térmicamente, PPD, es determinada con base

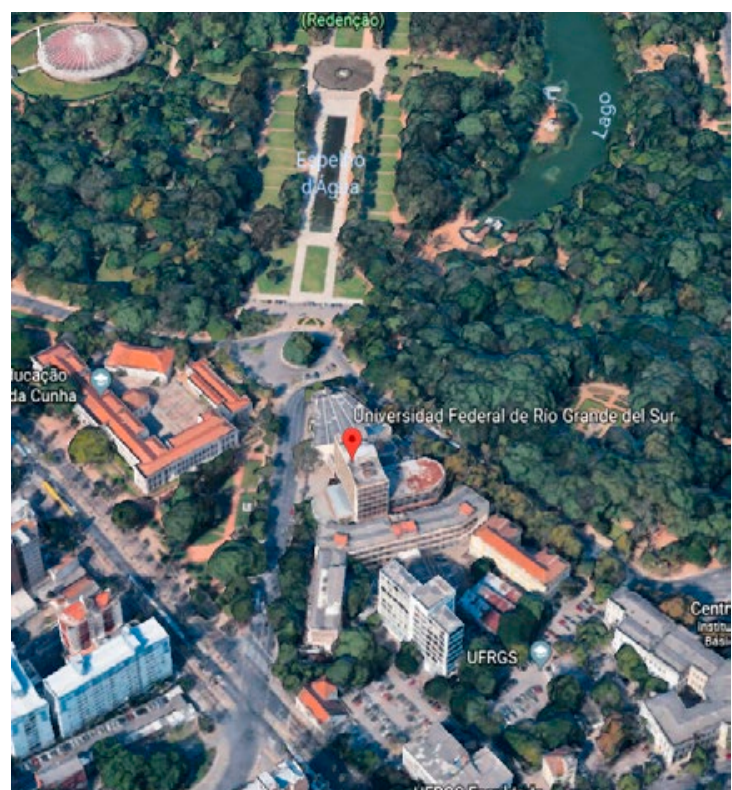

Universidad Federal de Rio Grande del Sur $U F R G S$, en el barrio de Agronomía de la ciudad de Puerto Alegre

METODOLOGIA PARA EL CÁLCULO DEL COMPORTAMIENTOTERMICO.

Las etapas a seguir en este trabajo son las siguientes:

1. Estudio de la casa a simular, dimensiones, materiales, orientación, etc.

2. Simulación con utilización del software Energy Plus 8.2 (2014)

3. Comparación datos medidos y simulados, índices de confort y energía de climatización.

\section{Localización de la vivienda.}

La vivienda estudiada (prototipo Alvorada) se localiza en la entrada del Campus del Valle de la Universidad Federal de Río Grande del Sur - UFRGS, en el barrio de Agronomía de la ciudad de Puerto Alegre $\left(30^{\circ} 02^{\prime} \mathrm{S} ; 51^{\circ} 13^{\prime} \mathrm{W}\right)$, construida en el terreno perteneciente al Laboratorio de Energía Solar. 
Características de la construcción.

El prototipo Alvorada posee un área total aproximada de $48 \mathrm{~m}^{2}$ los cuales se distribuyen de la siguiente forma: dos dormitorios, una sala de estar integrada a la coci- na, un baño, una galería y una pequeña área de servicio descubierta. La disposición de los ambientes, dimensiones internas, alturas y aberturas son mostrados en un plano de planta y corte en la Fig. 1 .

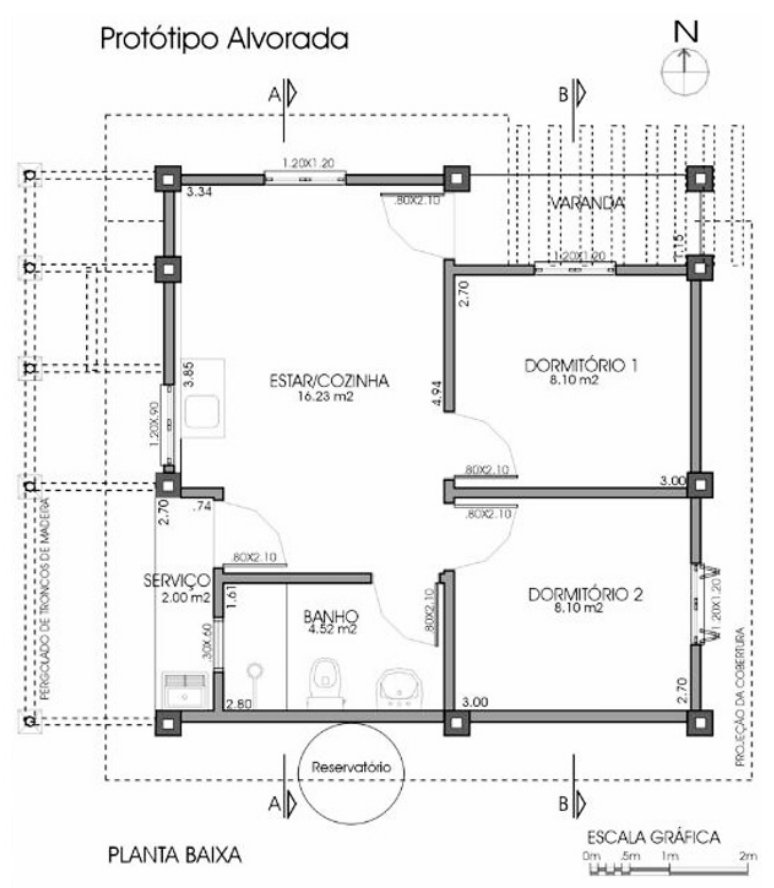

(a)

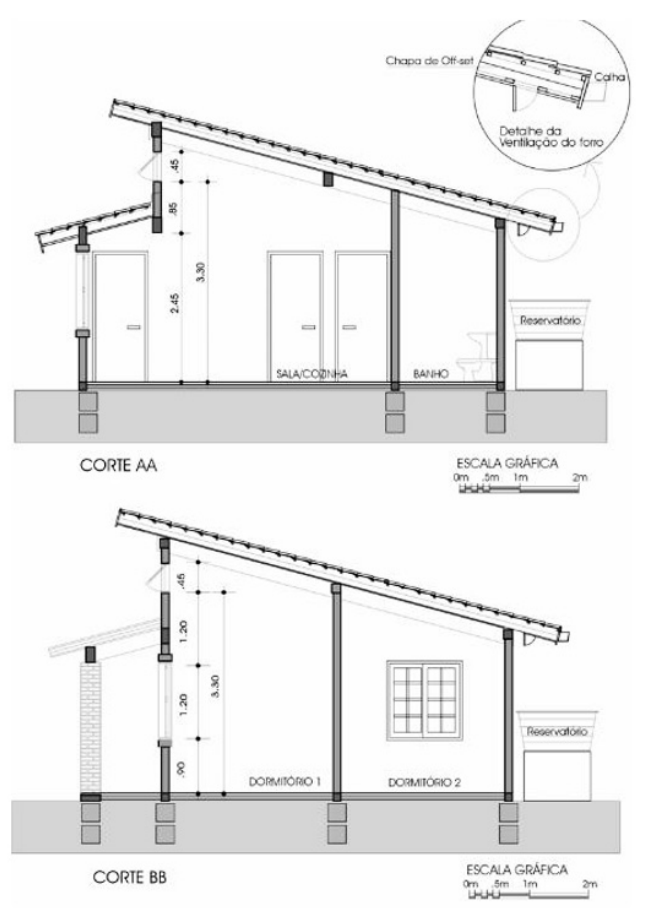

(b)

Figura 1: a) Plano de planta del prototipo Alborada, b) Cortes $A$-A y B-B presentados en la figura 2 a) (fuente: Morello, 2005).
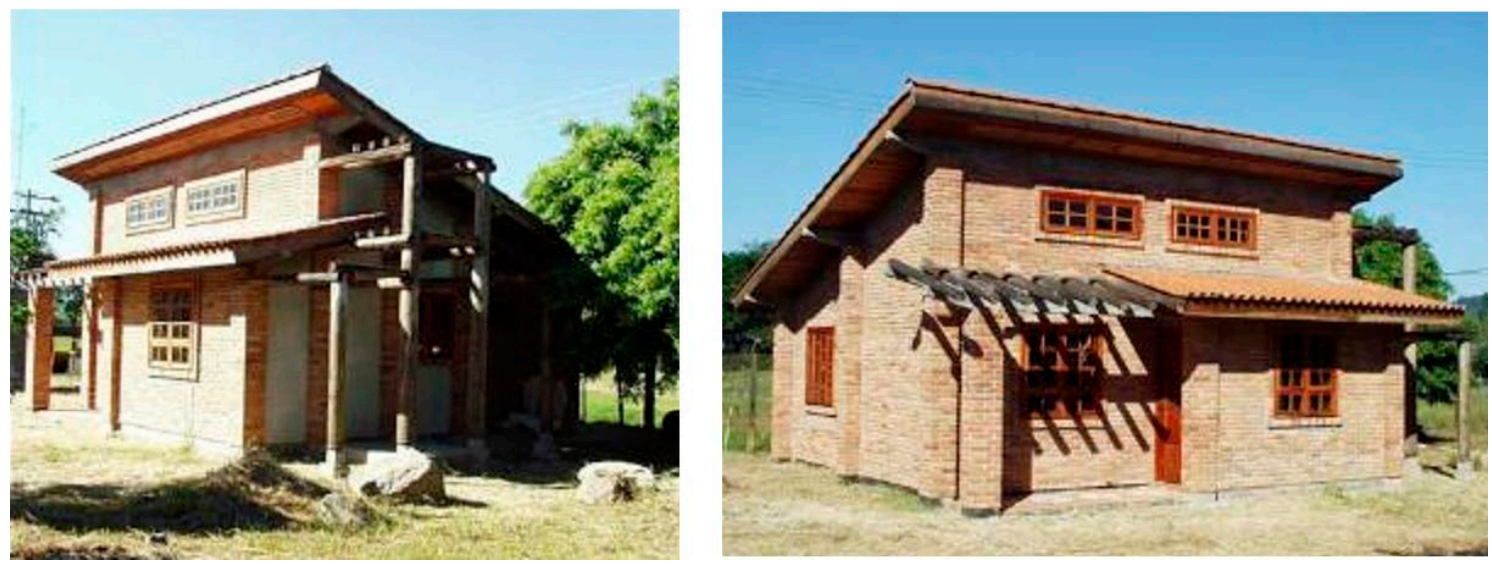

Figura 2: Fotografias del prototipo Alborada Este/Norte y Norte/Oeste. 
Características de los materiales y elementos componentes de la edificación.

Para poder simular el comportamiento térmico de la vivienda analizada es necesario contar con las características termo físicas de los materiales de construcción, de interés para el análisis del comportamiento térmico de la edificación. En la Tab. 1 son presentados los valores de absortancia $(\alpha)$ para radiación solar y emisividad $(\varepsilon)$ extraídos de la Norma ABNT (2005).

\begin{tabular}{|l|l|l|}
\hline Tipo de Superficie & Absortancia $(\alpha)$ & Emisividad $(\varepsilon)$ \\
\hline Concreto & $0,65 / 0,80$ & $0,85 / 0,95$ \\
\hline Ladrillo & $0,65 / 0,80$ & $0,85 / 0,95$ \\
\hline Teja & $0,75 / 0,80$ & $0,85 / 0,95$ \\
\hline Revoque & $0,30 / 0,50$ & $0,85 / 0,95$ \\
\hline Vidrio común & Transparente & $0,90 / 0,95$ \\
\hline
\end{tabular}

Tabla 1: características físicas de los materiales de construcción.

En la Tab. 2 se presentan las propieda- conductividad térmica $(\lambda)$ y calor especifico des térmicas de los materiales que compo- (c). En la Tab. 3 se presenta la transmitancia nen las paredes y el techo de la edificación. y capacidad térmica de los materiales utiliLos valores que se detallan son densidad $(\rho)$, zados en la construcción.

\begin{tabular}{|l|c|c|c|}
\hline Material & Densidad $\rho[\mathrm{kg} / \mathrm{m}]$ & Conductividad Térmica $\lambda[\mathrm{W} / \mathrm{m} . \mathrm{K}]$ & $\begin{array}{c}\text { Calor especifico c } \\
{[\mathrm{kJ} / \mathrm{kg} . \mathrm{K}]}\end{array}$ \\
\hline Concreto & 2400 & 1,75 & 1,00 \\
\hline Cerámica (ladrillos) & 1600 & 0,90 & 0,92 \\
\hline Mortero & 2000 & 1,15 & 1,00 \\
\hline Vidrio común & 2700 & 1,10 & 0,84 \\
\hline Cerámica (tejas) & 2000 & 1,05 & 0,92 \\
\hline Lámina de aluminio & 2700 & 230 & 0,88 \\
\hline Madera & 600 & 0,14 & 2,30 \\
\hline
\end{tabular}

Tabla 2: Propiedades térmicas de los materiales de construcción.

\begin{tabular}{|l|c|c|}
\hline Materiales y revestimiento & Transmitancia U $\left[\mathrm{W} / \mathrm{m}^{2} . \mathrm{K}\right]$ & $\begin{array}{c}\text { Capacidad CT } \\
{\left[\mathrm{KJ} /\left(\mathrm{m}^{2} . \mathrm{K}\right)\right]}\end{array}$ \\
\hline Ladrillo macizo $11 \mathrm{~cm}$ sin revoque & 3,49 & 170 \\
\hline $\begin{array}{l}\text { Ladrillo macizo } 22 \mathrm{~cm} \text { sin revoque (borde de } \\
\text { ventanas) }\end{array}$ & 2,47 & 337 \\
\hline $\begin{array}{l}\text { Ladrillo macizo } 11 \mathrm{~cm} \text { con revoque en un solo } \\
\text { lado }\end{array}$ & 3,29 & 210 \\
\hline $\begin{array}{l}\text { Ladrillo macizo } 2 * 11 \mathrm{~cm} \text { con revoque en un solo } \\
\text { lado }\end{array}$ & 2,33 & 401 \\
\hline
\end{tabular}




\begin{tabular}{|c|c|c|}
\hline $\begin{array}{l}\text { Ladrillo macizo } 11 \mathrm{~cm} \text { con revoque en ambos } \\
\text { lados }\end{array}$ & 3,11 & 251 \\
\hline Vigas de concreto $15 \mathrm{~cm}$ de espesor & 3,91 & 360 \\
\hline $\begin{array}{l}\text { Ladrillo } 11 \mathrm{~cm} \text { - aire } 13 \mathrm{~cm} \text { - ladrillo } 11 \mathrm{~cm} \mathrm{sin} \\
\text { revoque }\end{array}$ & 1,77 & 337 \\
\hline $\begin{array}{l}\text { Pilar de ladrillos } 22 \mathrm{~cm} \text { - revoque } 2 \mathrm{~cm} \text { - ladrillo } \\
11 \mathrm{~cm}\end{array}$ & 1,88 & 525 \\
\hline $\begin{array}{l}\text { Cubierta de tejas, lamina de aluminio y } \\
\text { revestimiento de madera }\end{array}$ & 1,11 & 32 \\
\hline
\end{tabular}

Tabla 3: Propiedades térmicas de los materiales de construcción.

Variables Térmicas.

Las variables utilizadas para la caracterización del prototipo Alvorada fueron las siguientes:

a) Temperatura: de globo $(\mathrm{Tg})$, de bulbo seco (Tbs), de bulbo húmedo (Tbh).

b) Humedad relativa del aire (HR).

\section{SIMULACIÓN CON ENERGY PLUS}

En la actualidad existen diversas aplicaciones de simulación para evaluar el comportamiento térmico de edificaciones; entre ellas encontramos herramientas como IESVE, IDA ICE, EDSL TAS, HAP, Equest, etc. Además, también se encuentran programas como Trnsys (complicado de utilizar para un usuario medio) y Ecotect (impreciso y sin evolución).

Sin embargo, ninguna de ellas está basada en un motor de simulación abierto, como EnergyPlus, que se destaca como uno de los motores de simulación más potentes y la apertura del código fuente del programa lo ha reforzado como la referencia a nivel mundial.

Conforme a Crawley et al, 2001, el EnergyPlus es un software relativamente nuevo, que comenzó a ser desarrollado en 1996 por un equipo formado por el Laboratorio de Investigación en Ingeniería de Construcción del Ejército de Estados Unidos (CERL), por la Universidad de Illinois (UI), el Laboratorio Nacional Lawrence Berkeley (LBNL), la Universidad del Estado de Oklahoma (OSU), Analitcs GARD, y el Departamento de Energía de Estados Unidos (DOE).

EnergyPlus se basa en las características más populares de otros dos programas de simulación energética en edificaciones, el Building Loads Analysis and System Thermodynamics, BLAST, desarrollado por el Departamento de Defensa de los Estados Unidos (DOD) y el Departamento de Defensa de Estados Unidos (DOD) el DOE-2, patrocinado por el Departamento de Energía de Estados Unidos (DOE).

Para simular en Energy Plus, tres componentes básicos forman el bloque principal de la aplicación; un gestor o controlador de simulación, un módulo de simulación del balance de calor y masa y un módulo de simulación de los sistemas de edición (Crawley et al, 2005). 
Bloque principal: Carga de datos para la simulación

En primer término, se define la localización donde se ubicará la vivienda a simular, seguidamente la cantidad de días a simular y se carga la base de datos climatológicos utilizado para la simulación.

A seguir se detallan los datos y parámetros que son seleccionado, diseñados y cargados en el Energy Plus para la simulación

\section{Cargar caracteristicas de materiales}

Se ingresan las características físicas y ópticas de los materiales con los que ha edificado el prototipo según los valores que fueron detallados en las Tablas 1,2 y 3.

Construcción física del modelo

Se crean los elementos que conforman la estructura física externa e interna de la vivienda: paredes, techo, piso, ventanas, etc., (Fig.3) y en cada una de ellas se definen las capas que conforman la construcción. Comenzando desde los materiales de las superficies exteriores hacia el interior, es decir, revoque, ladrillo, aislante, ladrillo y revoque. Seguidamente se definen las superficies de transferencia de calor según a la fuente que sea expuesta, solar, viento, etc., las sub-superficies (ventanas, puertas) y los muebles, arboles, aleros, etc. (Westphal, 2006).

En la Fig. 3 se presenta el diseño elemental del prototipo habitacional analizado, junto con su representación en el programa Sketchup.

Características de uso

Para realizar el estudio do confort térmico en la edificación presentada, se determinan diversas características de sus moradores; características definidas en intervalos horarios denominados schedules. Entre estas definiciones se pueden citar, rutinas de ocupación, nivel de actividad, factor de vestimenta y número de moradores. También se consideraron las ganancias internas debido a lámparas, equipamientos eléctricos y la renovación do aire.

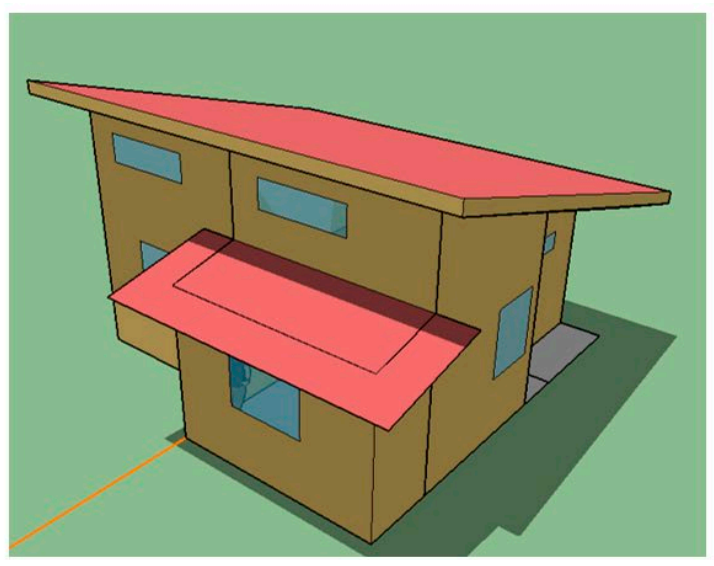

Figura 3: Diseño elemental del prototipo habitacional analizado, junto con su representación en el programa Sketchup que utiliza la herramienta de visualización OpenStudio del Energy Plus. 


\section{Carga Térmica}

La carga interna hace referencia a los tipos de fuente de calor existentes en la vivienda, como ser las luminarias, personas, equipamientos eléctricos, los cuales deben ser definidos por la potencia que ellos generan, sus características de emisión y los respectivos schedules antes creados. Para determinar los valores necesarios a introducir se utilizaron catálogos de lámparas fluorescentes da marca Philips, para los aparatos eléctricos se adoptan las potencias consignadas en catálogos de electrodomésticos y para el caso de la energía que las personas libera por su actividad metabólica según las normas ISO 7730.

\section{Infiltración de aire}

Debido a que a casa estaba cerrada los caudales de infiltración son bajos y se define como $0.03 \mathrm{~m}^{3} / \mathrm{s}$. Es importante tener en cuenta que para el caso de tener personas dentro se recomienda una infiltración de $0.75 \mathrm{~m}^{3} / \mathrm{s}$ por persona, y para ter como referencia se calculó que el valor de renovación para un aire acondicionado de $2000 \mathrm{Wh} y$ de 6500 Wh varían de $0.09 \mathrm{~m}^{3} / \mathrm{s}$ hasta 0.28 $\mathrm{m}^{3} / \mathrm{s}$ (Carrier, 1999).

\section{RESULTADOS}

Esta primera simulación fue con el objetivo de comparar los valores obtenidos por el Energy Plus en periodo de un año, considerando que no existen cargas térmicas producidas por personas y artefactos (casa vacía). El año de simulación utilizado en el Energy Plus fue el TMY - Typical Meteorological Year correspondiente al año 1954 en la ciudad de Porto Alegre (Cebecauer y
Suri, 2015).

En la Tab. 4 se presentan los valores medios mensuales de temperatura de bulbo seco ( $T b s)$ y humedad relativa que fueron introducidos en intervalos horarios en con el programa Energy Plus.

Es importante destacar que no se están comparando iguales valores exteriores ya que los TMY son un comportamiento medio y el año en cuestión puede diferir.

Los valores horarios obtenidos en la simulación son introducidos dentro de una carta psicométrica y comparados con la distribución que se obtiene con las mediciones horarias dentro de la casa. Para tener un primer análisis, y una idea general del comportamiento de la edificación, se confeccionaron las cartas bioclimáticas para el periodo completo en el software GERMET (software desarrollado por el GER-UNNE), que permite ver los valores horarios de Tbs y HR dentro de la

\begin{tabular}{|l|c|c|}
\hline \multicolumn{1}{|c|}{ Mes } & Tbs TMY $\left({ }^{\circ} \mathrm{C}\right)$ & HR TMY (\%) \\
\hline Enero & 24.6 & 80.0 \\
\hline Febrero & 24.3 & 81.6 \\
\hline Marzo & 23.4 & 80.9 \\
\hline Abril & 19.8 & 83.1 \\
\hline Mayo & 14.4 & 83.9 \\
\hline Junio & 14.9 & 89.6 \\
\hline Julio & 13.7 & 87.7 \\
\hline Agosto & 16.2 & 83.4 \\
\hline Septiembre & 16.9 & 86.0 \\
\hline Octubre & 18.3 & 81.5 \\
\hline Noviembre & 21.3 & 74.7 \\
\hline Diciembre & 23.2 & 71.7 \\
\hline Media & $\mathbf{1 9 . 3}$ & $\mathbf{8 2 . 0}$ \\
\hline
\end{tabular}

Tabla 3: Valores medios mensuales para los TMY de la ciudad de Porto Alegre para el año 1954. 
carta psicométrica para determinar en adecuadas de implementación de soluqué región de confort se encuentra las ciones para llevar a las personas que se personas dentro de la edificación analiza- encuentren en cualquier coordenada deda (Givoni, 1992). terminada por cualquier par de variables

En la Fig. 4 a) se presentan la car- a la zona de confort(zona n $\left.{ }^{\circ} 5\right)$. En la Fig. ta bioclimática desarrollado dentro del 5 b) se presenta la carta psicométrica con GER denominado GERMET con la los valores simulados para el interior de la descripción de las áreas y las estrategias vivienda.

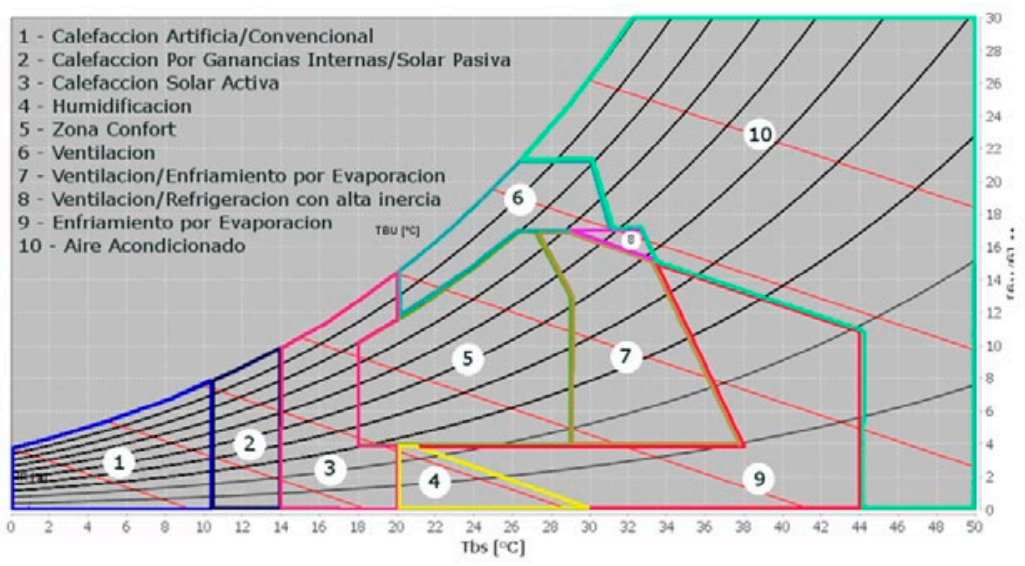

(a)

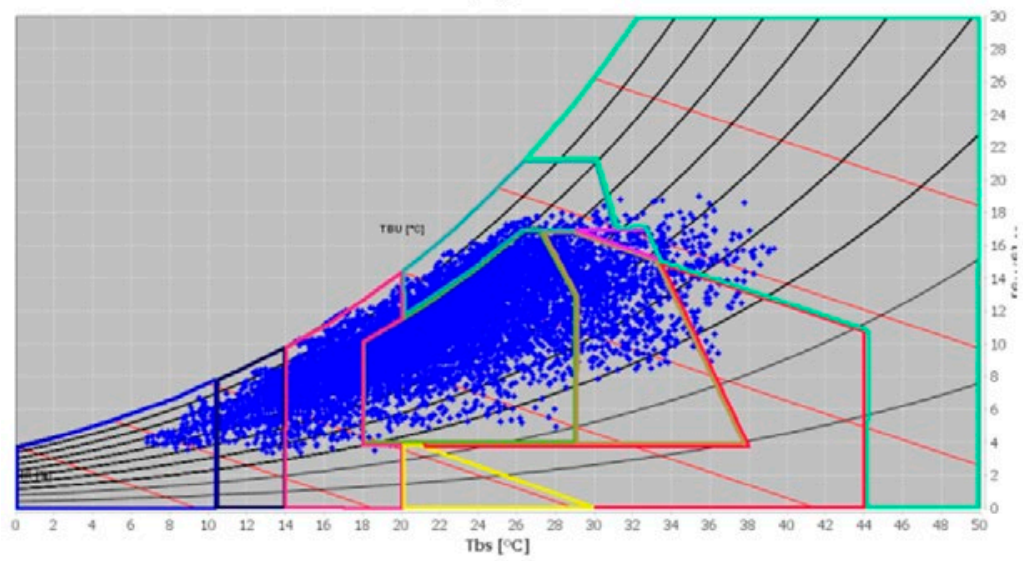

Figura 5: a) Diagrama Psicométrico con demarcación de las zonas que lo transforman en una Carta bioclimática obtenida (año 1954).b) Carta bioclimática obtenida con los datos internos de humedad y temperatura del aire para el archivo TMY de Porto Alegre (año 1954) obtenidos de simulación sin personas dentro de la vivienda.

Correlacionando los valores de tem- riodo estudiado. En la Fig. 5 b) se observa peratura y humedad relativa del aire exte- que los datos de Tbs y HR obtenidos en rior, las cartas bioclimáticas presentan la simulación horaria muestran que las perdistribución de Tbs y HR durante el pe- sonas a lo largo del año se encuentran en 
zonas fuera de la región de confort.

Realizando un análisis de la distribución de condiciones según el estado de confort o desconfort, ya sea por frio o calor, en primera instancia se denota que un $40.4 \%$ del tiempo las personas en el interior de la vivienda se encuentran en estado confort, mientras que para las condiciones externas el tiempo es de 22,5\%. Es decir, es necesario aplicar algún tipo de estrategia que permita llevar ese 59,6\% del tiempo de desconfort dentro de la vivienda a condiciones adecuadas.

En la Fig. 6 se presenta la variación de temperatura de bulbo seco interna y externa, así como la variación de humedad relativa porcentual interna y externa para el periodo simulado desde el día 19 al 27 de diciembre. También se presenta el comportamiento de la radiación solar para los mencionados días. En la Fig. 6 se observa que existe un desfasaje temporal entre la máxima irradiancia solar y la temperatura, así como la el efecto se observa que las variaciones internas tienen menor amplitud que las variaciones externas.

Correlacionando los valores de temperatura y humedad relativa del aire exterior, las cartas bioclimáticas presentan la distribución de Tbs y HR durante el periodo estudiado. En la Fig. 5 b) se observa que los datos de Tbs y HR obtenidos en simulación horaria muestran que las personas a lo largo del año se encuentran en zonas fuera de la región de confort.

Realizando un análisis de la distribución de condiciones según el estado de confort o desconfort, ya sea por frio o calor, en primera instancia se denota que un $40.4 \%$ del tiempo las personas en el interior de la vivienda se encuentran en estado confort, mientras que para las condiciones externas el tiempo es de 22,5\%. Es decir, es necesario aplicar algún tipo de estrategia que permita llevar ese 59,6\% del tiempo de desconfort dentro de la vivienda a condiciones adecuadas.

En la Fig. 6 se presenta la variación de temperatura de bulbo seco interna y externa, así como la variación de humedad relativa porcentual interna y externa para el periodo simulado desde el día 19 al 27 de diciembre. También se presenta el comportamiento de la radiación solar para los mencionados días. En la Fig. 6 se observa que existe un desfasaje temporal entre la máxima irradiancia solar y la temperatura, así como la el efecto se observa que las variaciones internas tienen menor amplitud que las variaciones externas.

Por lo expresado se hace necesario evaluar las intervenciones apropiadas para llevar la mayor cantidad de puntos que se encuentran fuera de la zona de confort, dentro de la misma. Se observa, de la Fig. 5 , que principalmente son 4 las estrategias necearías para llevar a que $90 \%$ del tiempo las personas que habitan esta casa se encuentren en una condición de confort. Las estrategias son: calefacción solar pasiva y activa, ventilación y refrigeración.

Se puede planear entonces: modificaciones infraestructurales o agregar equipos que entreguen o retiren energía térmica para mantener la condición interna 


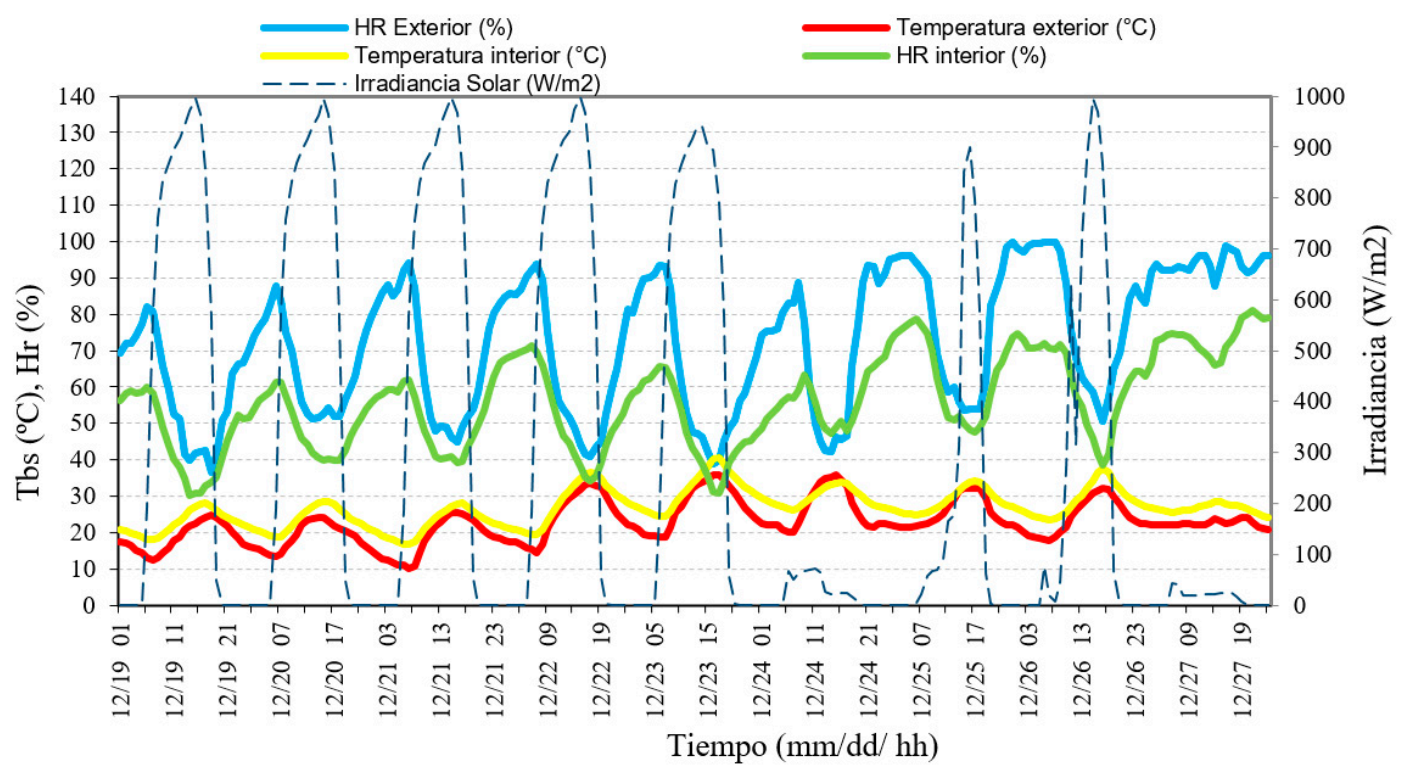

Figura 6: Valores horarios simulados de temperatura, irradiancia y humedad relativa a lo largo de 8 (ocho) dias del mes de diciembre para la vivienda cerrada y sin personas.

de confort. Para este primer estudio, que tiene como objetivo dimensionar el área acondicionado frio/calor más adecuado, se optara por calcular la energía necesaria y seleccionar el equipo que satisfaga las exigencias. Es importante denotar que el cálculo de PMV solo tiene sentido cuando existan personas dentro de la vivienda, por esta razón, la siguiente simulación se realiza definiendo una vivienda habitada.

Para cumplir lo planteado en el párrafo anterior se realiza una nueva simulación donde se agregan 4 (cuatro) personas que viven en el prototipo experimental y a cada una se de las personas se le asigna un cronograma de actividades, un valor de tasa metabólica, de ropa y tiempo dentro de la casa para el periodo de un año. Además, se agrega a la casa la carga térmica asociada a las luces y diferentes equipamientos del hogar con su correspondiente cronograma de encendido, apagado y potencia consumida, así como se ha modificado el grado de infiltración (aumentado respecto a una vivienda cerrada).

A través de la simulación realizada, con las definiciones mencionadas en el párrafo anterior, se obtuvieron los valores de PMV a lo largo del año. Como se ha mencionado el PMV es un paramento que varía ente 1 y -1 cuando las personas se encuentran en condiciones de confort. Los resultados presentados en la Fig. 7 muestran que existe un alto porcentaje de horas en el cual las personas se encuentran en condiciones de desconfort. Para solucionar esta situación se procede a calcular la energía necesaria de calefacción y de refrigeración.

Para calcular los requerimientos energéticos necesarios para estar en una situación de confort (PMV entre 1 y -1), 
que se lograría a través de una trasferencia energética de una cierta cantidad de aire frío o caliente, primero se reduce la infiltración siendo ella igual al valor mínimo de renovación de aire por persona, por el número de personas. Luego se define que el equipo encargado de entregar esa cantidad de energía trabaje según los valores de un termostato accionado por los valores de Tbs interior. Como se observa en la carta psicométrica la zona de confort varía según la estación entre $20^{\circ} \mathrm{C}$ y $28^{\circ} \mathrm{C}$ para una humedad relativa de 40 a $80 \%$. Para la simulación se utilizan estos límites para el termostato para, tomado los mismos como límites, se obtengan los valores de potencia térmica necesaria para el ambiente a lo largo del año. Es decir, con este paso es posible determinar la potencia del aire acondicionado frio/calor que permita mantener las condiciones de confort (PMV entre 1 y -1 ).

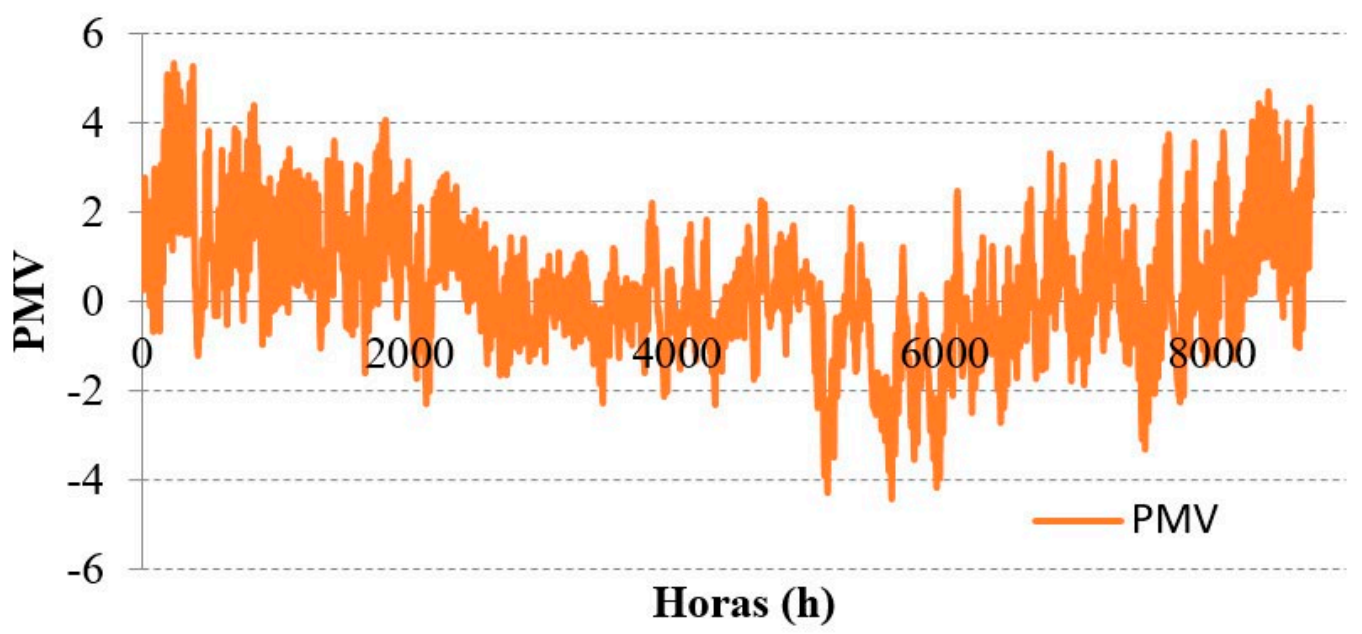

Figura 7. Variación del PMV a lo largo del año en la vivienda analizada.

En la Fig. 8 se presentan los valores de energía demandada para calefacción y refrigeración, se observa que la energía anual asociada a aplicaciones de refrigeración para llevar las condiciones interna a valores de confort son aproximadamente 3,5 mayores que las necesidades energéticas para calefacción.

Resultados obtenidos en la simulación horaria permiten determinar el día en el cual se produce la mayor carga de climatización. Específicamente se observa que la mayor carga de refrigeración se produce el día 16 de enero desde las 13:00 a las 14:00 horas, donde la energía necesaria llega a alcanzar el valor de 3,40 kWh. La máxima demanda de calefacción se produce en el día 30 de julio a las 6:00 am, y su valor es de 1,40 kWh.

Con los valores de demanda calculados es posible afirmar que la carga de refrigeración será la que definirá el dimensionamiento y selección del sistema de aire acondicionado frío/calor adecuado para la climatización del ambiente analizado. En este punto tenemos varias opciones co- 
merciales que responden adecuadamente al sistema de calefacción necesario que van desde los 3,0 a los 3,5 kW. Aquí es importante destacar que, el valor de $3,40 \mathrm{kWh}$ se presentó en un día y horario especifico, para le selección del aire se busca un sistema que responda al 99\% de las condiciones internas y un sistema tipo Split frio/calor de 3000 W responde adecuadamente a esta situación. Se obtiene que para aire acondicionados de esta potencia, clase A, el coeficiente de desempeño varía entre 3,0 y 3,4, por lo cual la energía eléctrica demandada será de aproximadamente $1000 \mathrm{~W}$.

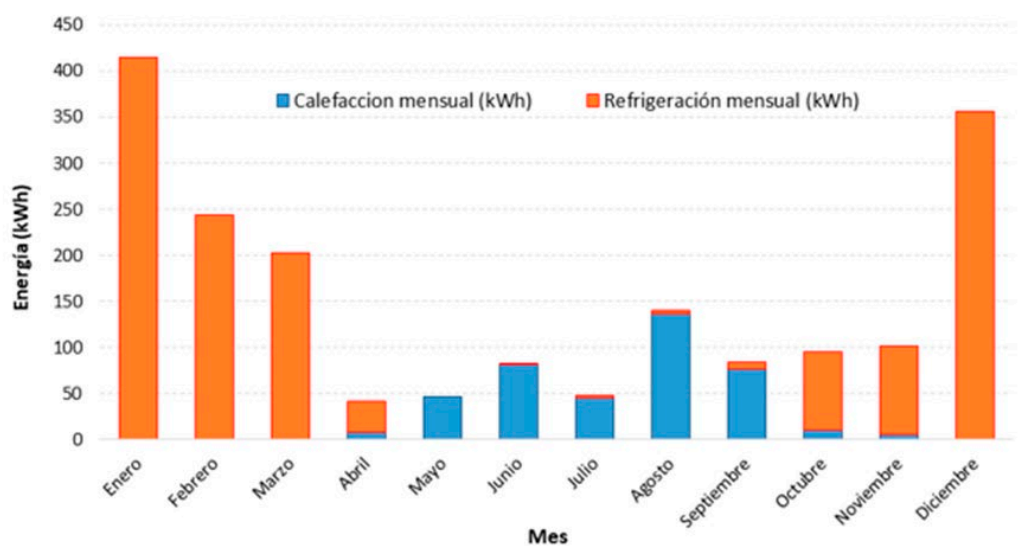

(a)

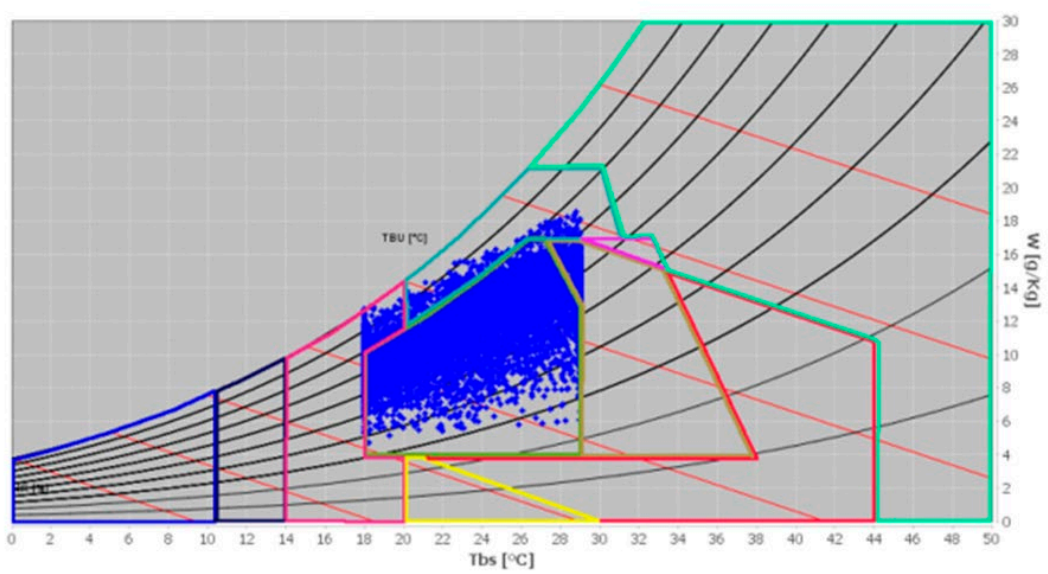

(b)

Figura 8: a) energía mensual necesaria para llevar las condiciones del ambiente analizado a condiciones de conforte térmico para las personas b) distribución de los valores de Tbs y HR\% dentro de la carta psicométrica luego de que se ha agregado el elemento termostato a la simulación.

En la Fig. 8 a) se presentan los resulta- lizado a condiciones de conforte térmico dos que muestran la energía necesaria para para las personas. En la Fig. 8 b) se presenllevar las condiciones del ambiente ana- ta como ha quedado la distribución de los 
valores de Tbs y HR\% dentro de la carta psicométrica luego de que se ha agregado el elemento termostato a la simulación. En esta última figura se observa que el elemento termostato se encarga de, a través de un aporte energético, llevar las condiciones (puntos) que se encuentra fuera de la zona $\mathrm{n}^{\circ} 5$ a la región de confort.

\section{CONCLUSION}

En este trabajo se determinó el comportamiento térmico del prototipo habitacional localizado en el Campus del Valle de la UFRGS - Universidade Federal do Rio Grande do Sul en la ciudad de Porto Alegre, a través de una simulación realizada con el programa computacional Energy Plus. Los valores obtenidos a lo largo de un año de simulación fueron comparados estadísticamente y presentados gráficamente en una carta psicométrica para determinar las mejores estrategias de confort. Se determinaron las diferencias existentes entre los valores internos y externos de los principales parámetros climáticos para una vivienda cerrada y vacía un año completo, inicialmente. Seguidamente se simuló la vivienda agregando personas y equipamiento eléctrico para estudiar las condiciones de confort (PMV) para una vivienda con 4 personas. Esta simulación permitió determinar el índice denominado PMV, propuesto por Fanger, para evaluar las condiciones de confort. Lo que resultó en un elevado número de horas en que las personas se encuentran en condiciones de desconfort.

Se calculó la energía necesaria para llevar el ambiente interno del prototipo habitacional a condiciones de confort, una nueva simulación horaria determinó que la carga por refrigeración máxima es de 3,5 kWh para el día 16 de enero, y la demanda por refrigeración es 3,5 veces superior a la de calefacción. Con estos datos se determinó la potencia más adecuada para un sistema de climatización frio/calor. De esta forma, se calculó que con un aire acondicionado de $3000 \mathrm{~W}$, clase A, con un COP de 3 , se responde adecuadamente al 99\% de las condiciones anuales que necesiten un apoyo energético para llevar los ambientes de la vivienda, habitada por 4 personas, a condiciones de confort térmico.

Este trabajo presentó la metodología de utilización de una herramienta computacional sobre un caso práctico, la simulación otorga una gran flexibilidad de evaluar diferentes condiciones y recursos energéticos necesarios hasta optimizar el diseño estructural o la cantidad de energía que se debe entregar para acondicionar adecuadamente una vivienda. De esta forma, con implementaciones de arquitectura bioclimática y análisis de equipamientos de bajo consumo es posible determinar la relación costo inicial versus demanda de energía anual más apropiada. 


\section{REFERENCIAS}

ABNT - Associação Brasileira de Normas Técnicas (2005 ) - Desempenho térmico de edificações: Parte 2: métodos de cálculo de transmitância térmica, da capacidade térmica, do atraso térmico e do fator de calor solar de elementos e componentes de edificações. Rio de Janeiro: ABNT, 27p.

Carrier Air Conditionung Company (1999). Manual de aire acondicionado. Editorial: Marcombo

Cebecauer T y Suri M (2015). Typical Meteorological Year Data: SolarGIS Approach. Energy Procedia. Volume 69. Pages 1958-1969

Crawley D; Hand J, Kummert M; Griffith B (2005). Contrasting the Capabilities of Building Energy Simulation Software tools. Energy Performance Simulation Software tools, U.S. Department of Energy, Energy Systems Research Unit, University of Wisconson-Madison, National Renewable Energy Laboratory.

Crawley, D.B., et al. (2001), EnergyPlus: creating a new-generation building energy simulation program. Energy and Buildings,. 33(4): p. 319-331. EnergyPlus 8.2, (2014). Building Energy Simula- tion. Developed in collaboration with NREL, various DOE National Laboratories, academic institutions, and private firms. https://energyplus.net/

Givoni, B (1992). Comfort, climate analysis and building design guidelines. Energy and Buildings, vol. 18, p. 11-23.

ISO 7730 (2006). Ergonomía del ambiente térmico. Determinación analítica e interpretación del bienestar térmico mediante el cálculo de los índices PMV y PPD y los criterios de bienestar térmico local.

Morello, A (2005). Avaliação doncomportamento térmico do protótipo habitacional Alvorada. Dissertação (Mestrado em Engenharia Civil). Universidade Federal do Rio Grande do Sul. Porto Alegre.

Westphal, Fernando Simon (2006). Curso: Introdução ao Energy Plus. Universidade Federal de Santa Catarina. Centro Tecnologico - Departamento de Engenharia Civil. Florianópolis. Brasil. Junho 2006. 